\title{
Une approche unifiée pour proposer des activités interactives entièrement personnalisées sur les profils d'apprenants
}

\author{
Blandine Ginon, Stéphanie Jean-Daubias \\ Université de Lyon, CNRS - Université Lyon 1, LIRIS, UMR5205, F-69622, France \\ blandine.ginon@liris.cnrs.fr, stephanie.jean-daubias@liris.cnrs.fr
}

\begin{abstract}
RÉSUMÉ. Dans le domaine des EIAH (Environnements Informatiques pour l'Apprentissage Humain), la personnalisation de l'enseignement tient un rôle majeur en raison $d u$ fort potentiel de ce paradigme pour l'apprentissage. Par ailleurs, dans le contexte des recherches sur le modèle ouvert de l'apprenant, de plus en plus d'EIAH proposent des activités réflexives basées sur la visualisation de profils d'apprenants. Néanmoins, la personnalisation de telles activités reste peu étudiée alors qu'elle pourrait rendre ces activités plus efficaces encore. Nous nous sommes intéressées à cette question et avons proposé une approche de personnalisation unifiée multi-aspects des activités sur les profils. Nous présentons dans cet article les modèles sur lesquels repose notre approche. Ces modèles permettent la création de séances d'activités sur les profils, de manière entièrement personnalisée par l'enseignant, tout en prenant en compte les connaissances et compétences des apprenants, ainsi que les capacités et préférences de l'ensemble des utilisateurs.
\end{abstract}

ABSTRACT. In the field of ILE (Interactive Learning Environments), personalization of education plays a major role because of the high potential of this paradigm for learning. Moreover, in the context of research on the open learner model, more and more ILE offer reflexive activities based on the visualization of learner profiles. However, the personalization of such activities is still poorly studied when it could make these activities more effective. We are interested in this issue and have proposed a unified approach of multiaspects personalization of activities on profiles. We present here the models underpinning our approach. These models enable the creation of sessions of activity on profiles, in a way completely customized by the teacher, taking into account the knowledge and skills of learners, and the capabilities and preferences of all users.

MOTS-CLÉS : profils, visualisation, personnalisation, modèles, Environnements Informatiques pour l'Apprentissage Humain

KEYWORDS: profiles, visualization, customization, models, Interactive Learning Environments

Revue Technique et Science Informatique. Volume $X-n^{\circ} \mathrm{x} /$ année, pages 1 à $X$ 


\section{Introduction}

Les profils d'apprenants sont au cœur des recherches en EIAH (Environnements Informatiques pour l'Apprentissage Humain). Deux termes sont employés pour désigner la représentation des informations concernant un apprenant, c'est-à-dire un humain en situation d'apprentissage (élève, étudiant, adulte en formation...): modèle ou profil. Si la distinction est parfois floue, nous préférons, comme (Vassileva et al., 2003) et (Keenoy et al., 2004) le terme de profil qui fait référence aux informations spécifiques à un apprenant donné, au terme de modèle qui englobe parfois le module qui gère ces données, voire le processus d'identification de ces données. Un profil d'apprenant rassemble des informations concernant un apprenant, collectées ou déduites à l'issue d'une activité pédagogique qui peut être papiercrayon (comme une interrogation écrite) ou logicielle (comme une séance d'utilisation d'un logiciel éducatif). Plus spécifiquement, le profil d'apprentissage d'un apprenant concerne ses connaissances et compétences, comme son niveau d'anglais ou son taux de réussite à un exercice de géographie. Un exemple volontairement simple de profil d'apprentissage est donné en Figure 1. Il s'agit d'un profil «papier-crayon » sous la forme la plus couramment utilisée dans les pratiques des enseignants : un bulletin de notes. Quant au profil de capacités d'un apprenant, il concerne ses capacités physiques et mentales, comme son niveau d'audition ou la durée moyenne de concentration qu'il est capable de fournir sur une activité pédagogique (Jean-Daubias, 2011).

\begin{tabular}{|c|l|l|l|}
\hline Prénom & Date & Épreuve écrite & Épreuve pratique \\
\hline \multirow{3}{*}{ Théo } & $11 / 03 / 11$ & $\begin{array}{l}\text { Tu fais des efforts mais c'est encore insuffisant } \\
\text { Restitution : 3/8 } \\
\text { Raisonnement : Extraction : 2/3 } \\
\text { Résolution : 3,5/5 }\end{array}$ & $\begin{array}{l}\text { Matériel : B } \\
\text { Consignes : B } \\
\text { Rendu : C }\end{array}$ \\
\cline { 2 - 5 } & $17 / 04 / 11$ & $\begin{array}{l}\text { Tu aurais obtenu la moyenne à cette épreuve } \\
\text { en apprenant le cours ! } \\
\text { Restitution : 2/8 } \\
\text { Raisonnement : Extraction : 2,5/3 } \\
\text { Résolution : 3/5 }\end{array}$ & \\
\hline
\end{tabular}

Figure 1. Profil d'apprentissage de Théo

Afin d'expliquer notre approche générale, nous nous appuyons sur le modèle ACUTE4Profiles qui détaille le cycle de vie des profils d'apprenants (cf. Figure 2) depuis les activités pédagogiques jusqu'à la proposition d'activités pédagogiques personnalisées en fonction du profil de l'apprenant (Jean-Daubias, 2011).

Si plusieurs points d'entrée sont possibles, commençons classiquement par les activités pédagogiques initiales (A) qui peuvent donner lieu à des traces. Ces traces permettent de constituer $(C)$ des profils d'apprenants initiaux grâce au processus de diagnostic des connaissances. Ces profils peuvent ensuite être uniformisés (U) en vue d'être intégrés à un système autre que celui qui est à l'origine de leur création. Les profils uniformisés peuvent être transformés (T) si besoin, et les profils 
transformés peuvent enfin être exploités (E). Parmi les exploitations possibles, figurent les activités sur les profils et la définition d'activités pédagogiques personnalisées en fonction du profil des apprenants. La définition d'activités pédagogiques conduit à la création de configurations, qui alimenteront les activités pédagogiques tracées $(\mathrm{A})$.

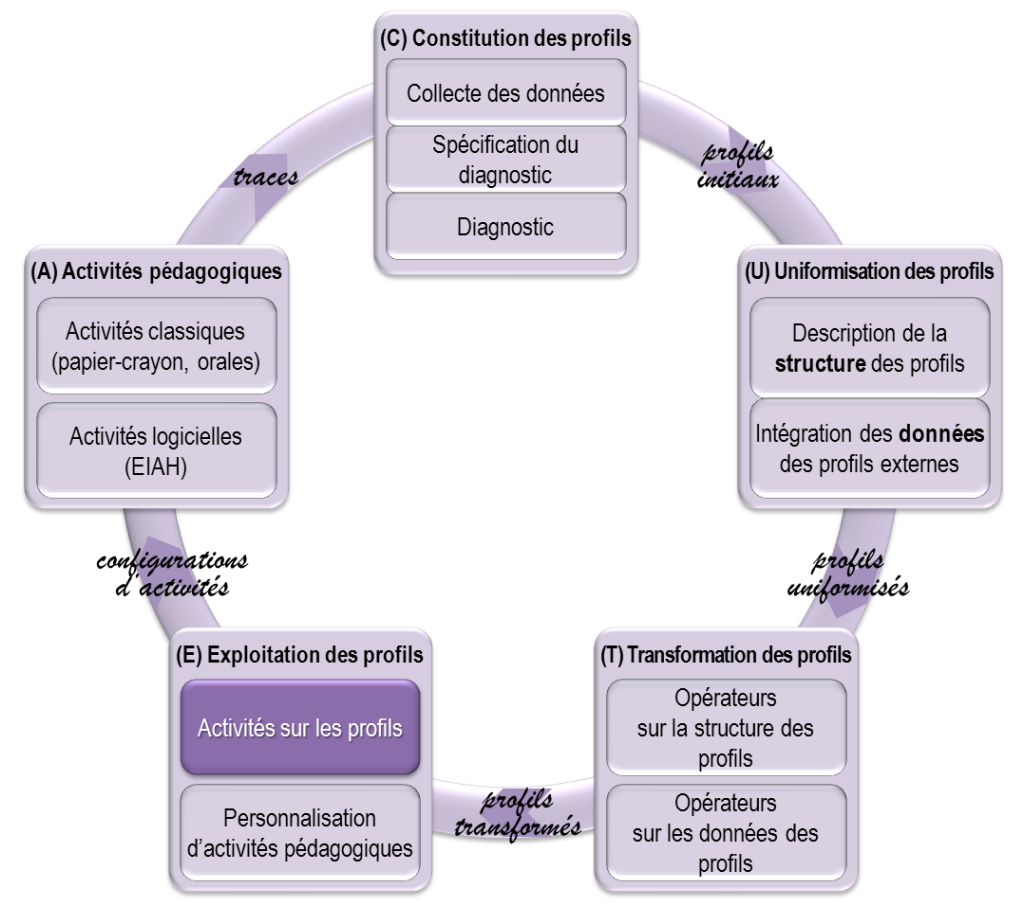

Figure 2. ACUTE4Profiles : modèle du cycle de vie des profils d'apprenants

Dans cet article, nous nous concentrons sur les activités sur les profils. Nous avons proposé une typologie de telles activités qui met notamment en évidence différents types d'activités sur les profils : consultation et comparaison de profils, positionnement par rapport aux pairs, appropriation des données du profil et intervention sur les profils (Jean-Daubias, 2011). Un enseignant de primaire peut par exemple faire travailler ses élèves sur les compétences en jeu pour le semestre en insistant sur les points positifs pour les élèves en difficulté et sur les points à améliorer pour les plus forts, puis définir avec eux des objectifs pour le semestre à venir. Dans un tout autre contexte, un organisme de formation professionnelle peut faire réaliser un bilan de fin de formation à ses stagiaires, afin de comparer compétences initiales et compétences finales.

Nous proposons plusieurs modèles complémentaires afin de permettre la personnalisation des activités sur les profils. Nous présenterons tout d'abord le contexte de nos travaux de recherche, en présentant en particulier les modèles PMDLe et PERSUMAP nécessaires à la compréhension des modèles qui font l'objet 
de cet article. Nous détaillerons rPMDLe et vPMDLe, nos modèles qui permettent de définir des représentations d'éléments de profils et des vues sur profils. Nous présenterons ensuite les évaluations que nous avons réalisées, avant de terminer par une discussion.

\section{Contexte de la recherche}

Cette recherche s'inscrit dans le cadre du projet PERLEA qui associe apports théoriques et propositions opérationnelles. Ce projet vise à proposer des modèles génériques et des outils unifiés pour une réutilisation et une exploitation mutualisée de profils d'apprenants hétérogènes, existants ou à venir, papier-crayon ou logiciels, dans des contextes différents et par des acteurs autres que leur auteur (Jean-Daubias et al., 2009). Dans cette partie, nous présentons les éléments de ce projet nécessaires à la compréhension de cet article : le langage de modélisation de profils PMDLe, le modèle de personnalisation des activités sur les profils PERSUMAP et l'environnement EPROFILEA qui met en œuvre ces modèles.

\subsection{Le projet PERLEA}

Depuis (Self, 1990), on sait que les profils d'apprenants peuvent être utilisés dans de nombreuses situations, et de nombreux logiciels pédagogiques établissent et exploitent des profils d'apprenants. Malgré l'intérêt pédagogique clairement démontré des activités sur les profils d'une part et de la personnalisation de l'enseignement d'autre part, il existe très peu de travaux concernant des activités sur les profils d'apprenants qui seraient variées, interactives, mais également personnalisées pour s'adapter à chaque utilisateur.

Le projet PERLEA vise à combler ce manque en adoptant une démarche générique conduisant à des outils unifiés. Sa problématique générale est la suivante : Comment permettre à des acteurs, éventuellement différents de leurs créateurs, de réutiliser des profils d'apprenants dans le but de proposer des exploitations mutualisées des profils à travers des activités personnalisées, adaptées à la fois aux besoins des enseignants et à ceux des apprenants, dans une démarche générique permettant de considérer différents niveaux scolaires et universitaires, ainsi que les disciplines les plus variées?

\subsection{Le langage de modélisation de profils d'apprenants PMDLe}

Pour ce travail de recherche centré sur les activités sur les profils d'apprenants, nous avons fait le choix de nous appuyer sur un langage de modélisation de profils, suffisamment générique pour permettre l'expression de profils variés, et adapté aux pratiques des enseignants. Nous utilisons le langage de modélisation de profil PMDLe (Jean-Daubias et al., 2009) (Ginon et Jean-Daubias, 2011), associé au projet 
PERLEA. L'opérationnalisation de PMDLe permet à un concepteur pédagogique de créer des modèles de profils qui seront par la suite instanciés par l'agent établissant le diagnostic sous forme d'autant de profils que d'apprenants, profils dont la structure sera commune, mais qui contiendront des données propres à chaque apprenant. La généricité de PMDLe permet de décrire des profils adaptés aux besoins spécifiques des enseignants les plus variés, à tous les niveaux d'enseignement, mais aussi de modéliser des profils d'apprenants issus de logiciels pédagogiques variés.

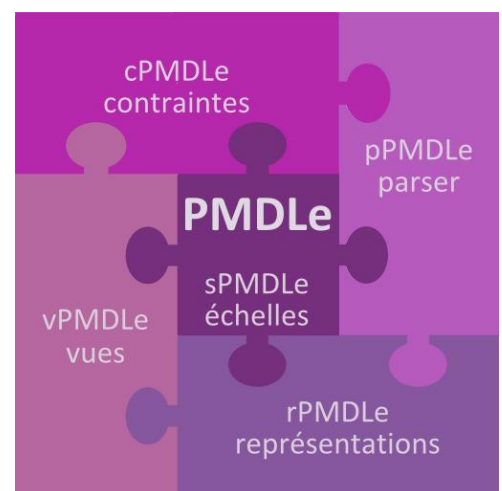

Figure 3. Représentation schématique de PMDLe et de ses extensions

PMDLe a été étendu par un ensemble de modèles qui peuvent être représentés comme les pièces d'un puzzle, cf. Figure 3. Au cœur de ce puzzle se trouve le langage de modélisation de profils PMDLe, indissociable de sPMDLe qui permet de définir des échelles numériques (comme une note de -5 à 5 ou un pourcentage) ou textuelles (comme acquis/ non acquis ou assez bien/ bien/ très bien) pour les éléments des profils PMDLe. Les modèles de définition de représentations d'éléments rPMDLe et de vues sur profils vPMDLe seront présentés en détail respectivement en sections 3.2 et 3.3 puisqu'ils sont au cœur de nos travaux de recherche sur les activités de visualisation de profils. Nous présenterons également en section 3.1 le modèle de contraintes sur profils cPMDLe que nous avons exploité dans cette recherche.

À titre d'exemple, la Figure 4 représente le profil de Théo, écrit en PMDLe. Les données spécifiques à Théo sont matérialisées par un fond gris, alors que la partie sur fond blanc correspond à la structure de profils commune notamment à tous les élèves de sa classe. Un profil PMDLe est composé d'un ensemble d'éléments, précisés en composantes et sous-composantes. Le profil de Théo est ainsi composé de deux éléments : un élément Épreuve écrite (cf. (1) sur la Figure 4) et un élément Épreuve pratique (cf. (2) sur la Figure 4), chacun constitué de composantes et souscomposantes.

Dans la partie «données» des profils PMDLe, les commentaires et les évaluations propres à un apprenant sont associés à une source et à une date. Ainsi, le commentaire de Théo pour le bac blanc 1 du 11/03/11 est « Tu fais des efforts mais c'est encore insuffisant. » (cf. (3) sur la Figure 4). Par ailleurs, les profils PMDLe 
prennent en compte l'évolution des connaissances des apprenants : ils peuvent contenir des informations relatives à plusieurs périodes d'évaluation pour un même élément, ce qui permet de refléter le niveau actuel d'un apprenant, sans perdre d'information sur son niveau passé. On constate ainsi que Théo avait obtenu 3 points lors du bac blanc 1 du 11/03/11 pour la composante Restitution des connaissances de la brique Épreuve écrite, alors qu'il n'a plus que 2 points pour le bac blanc $2 \mathrm{du}$ 17/04/11 (cf. (4) et (5) sur la Figure 4).

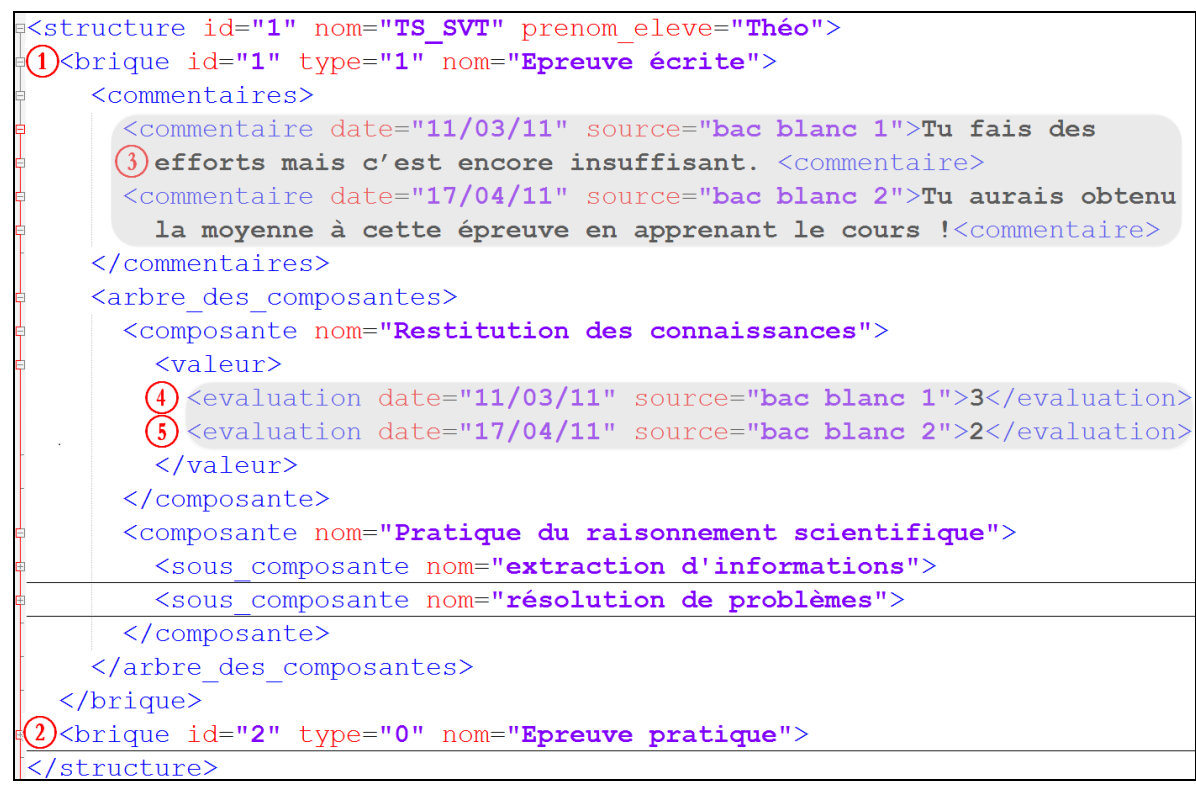

Figure 4. Profil de Théo décrit en PMDLe

\subsection{Le modèle de personnalisation unifiée multi-aspects des activités sur les profils PERSUMAP}

Dans le cadre du projet PERLEA, nous avons proposé un modèle de personnalisation des activités sur les profils d'apprenants. Le modèle PERSUMAP (PERSonnalisation Unifiée Multi-aspects des Activités sur les Profils) permet à la fois de définir des règles de personnalisation et de mettre en œuvre cette personnalisation (Ginon et Jean-Daubias, 2011). Il donne à l'enseignant la possibilité de personnaliser les activités sur les profils par la création de séances d'activités pour chaque type d'utilisateurs. Une séance d'activités est associée à une structure de profils décrite dans le langage PMDLe et concerne un type d'utilisateurs donné. Une séance d'activités associe aux activités sur les profils une vue sur profils respectant vPMDLe, le modèle de définition de vues sur profils, et une condition créée par combinaison de contraintes respectant le modèle de contraintes sur profils cPMDLe. Une vue sur profils associe à chaque élément d'un profil, un ensemble de 
représentations respectant le modèle de représentations d'éléments rPMDLe, et une condition respectant cPMDLe. L'application des règles définies par l'enseignant dans une séance d'activités permet de personnaliser les activités en fonction des connaissances et compétences d'un apprenant, et des capacités de l'utilisateur. Notons que dans le cas où un apprenant effectue une activité sur son profil, l'utilisateur et l'apprenant ne font qu'un.

Le modèle PERSUMAP permet également la prise en compte des préférences des utilisateurs. Le système exploite les possibilités du modèle en proposant à chaque utilisateur des représentations adaptées à ses préférences et respectant les choix établis par l'enseignant. L'utilisateur peut également modifier cette personnalisation, en sélectionnant d'autres représentations parmi celles disponibles, toujours de façon cohérente avec les choix de l'enseignant.

\subsection{Mise en ouvre dans l'environnement EPROFILEA}

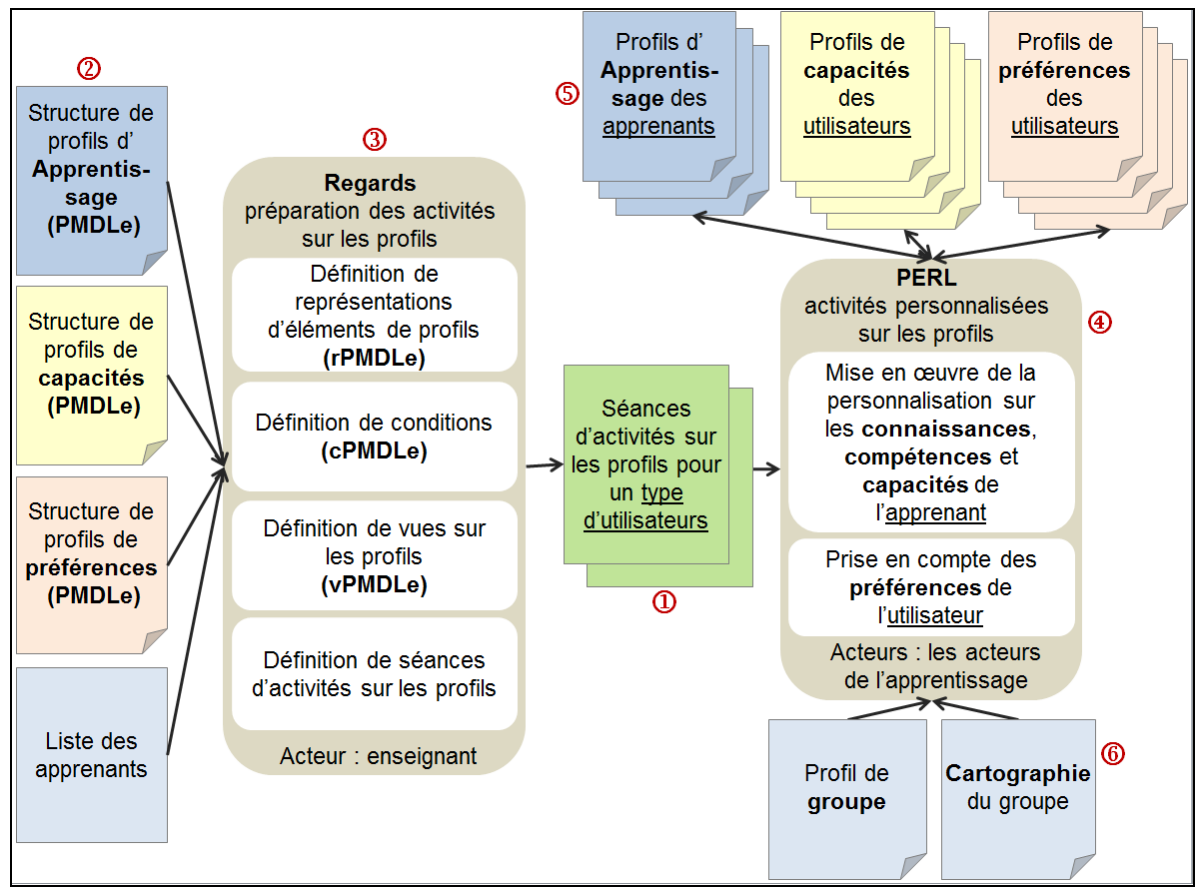

Figure 5. Architecture des activités sur les profils dans l'environnement EPROFILEA

Les différentes propositions théoriques faites au sein du projet PERLEA ont été mises en œuvre dans l'environnement informatique EPROFILEA. EPROFILEA s'adresse principalement à des enseignants et est utilisable dans différents niveaux d'étude et dans les matières les plus variées. Le processus de gestion de profils qu'il met en œuvre comporte deux phases. La phase de préparation permet d'obtenir des profils 
d'apprenants décrits selon un formalisme commun : le langage PMDLe. Un module permet notamment d'intégrer automatiquement dans un profil respectant PMDLe des données issues de profils respectant un formalisme spécifique à un autre EIAH, ou un standard donné, comme IMS-LIP (Lefevre et Jean-Daubias, 2012). Les profils de l'environnement peuvent ensuite être exploités dans la phase d'exploitation des profils. Dans l'environnement EPROFILEA, ces exploitations sont de deux types : d'une part la personnalisation d'activités pédagogiques basées sur le profil des apprenants est proposée dans le module Adapte (Lefevre et al., 2011) et, d'autre part, des activités interactives et personnalisées autour de la visualisation des profils d'apprenants sont proposées par les modules Regards et Perl (Ginon et JeanDaubias, 2011). Les propositions théoriques qui font l'objet de cet article sont mises en œuvre dans ces deux derniers modules.

Le module Regards permet à l'enseignant de préparer des séances d'activités sur les profils pour chaque type d'utilisateurs (apprenants, enseignants, familles d'apprenants, chercheurs, institutions...) (cf. (1) sur la Figure 5). Les séances d'activités sur les profils issues de Regards sont adaptées à une structure de profils d'apprentissage, à une structure de profils de capacités et à une structure de profils de préférences, toutes trois respectant PMDLe, ainsi qu'à une liste d'apprenants (cf. (2) sur la Figure 5). Pour créer des séances d'activités dans le module Regards (cf. (3) sur la Figure 5), l'enseignant peut utiliser les représentations d'éléments existantes respectant rPMDLe, ou en créer de nouvelles. Il peut également créer des contraintes sur profils respectant cPMDLe et portant sur le profil d'apprentissage, le profil de capacités ou la liste des apprenants; l'enseignant peut ensuite combiner ces contraintes pour former des conditions qui lui permettront par la suite de définir la personnalisation des vues sur profils ou des séances d'activités sur les profils en fonction des connaissances et compétences de l'apprenant et des capacités de l'utilisateur. Le module Regards permet également à l'enseignant de créer des vues sur profils vPMDLe, qui utilisent des représentations d'éléments et éventuellement des conditions. Après avoir défini les vues sur profils qu'il souhaite utiliser, l'enseignant peut enfin créer une séance d'activités sur les profils pour un type d'utilisateurs.

Ce travail de préparation de séances d'activités peut être long, en particulier lorsque l'enseignant désire créer de nouvelles représentations. Néanmoins, une fois les représentations créées, l'enseignant pourra les réutiliser pour une autre séance d'activités, y compris pour une séance d'activités portant sur une autre structure de profils. De plus, l'enseignant pourra facilement modifier une séance d'activités ou une vue, éventuellement en ajoutant ou modifiant certaines conditions, afin de ne pas repartir de zéro pour une prochaine utilisation. Dans la plupart des cas, l'enseignant pourra réutiliser ce travail de préparation quasi intégralement d'une année sur l'autre. Enfin, le système peut être fourni aux enseignants avec un ensemble de représentations correspondant aux usages fréquents.

Le module Perl (cf. (4) sur la Figure 5) quant à lui fournit à chaque utilisateur des activités sur les profils dont la personnalisation a été préalablement définie par 
l'enseignant à l'aide des différents modèles présentés dans cet article, dans les séances d'activités sur les profils issues de Regards (cf. (1) sur la Figure 5). La personnalisation porte d'une part sur les connaissances et compétences de l'apprenant dont le profil est au cœur d'une activité, ainsi que sur les capacités de l'utilisateur, et d'autre part sur les préférences de cet utilisateur. Pour cela, le module Perl utilise le profil d'apprentissage des apprenants concernés par chaque activité, ainsi que le profil de capacités et de préférences de l'utilisateur qui effectue l'activité (cf. (5) sur la Figure 5). Selon l'activité effectuée, Perl peut également faire appel au profil du groupe ou à la cartographie du groupe (cf. (6) sur la Figure 5), par exemple pour une activité de comparaison entre le profil d'un apprenant et le profil du groupe, ou pour une activité de positionnement de l'apprenant dans la cartographie du groupe.

\section{Des modèles pour la personnalisation d'activités sur des profils PMDLe}

Nous présentons dans cette partie les modèles nécessaires à l'exploitation de notre modèle de personnalisation PERSUMAP: modèles de définition de contraintes sur profils, de représentations d'éléments d'un profil, de vues sur profils et enfin de séances d'activités sur les profils d'apprenants.

\subsection{Exploitation du modèle de contraintes sur profils cPMDLe}

Pour permettre une personnalisation des activités sur les profils pour chaque utilisateur, il est nécessaire de spécifier dans quels cas un utilisateur accèdera à une activité ou dans quels cas il accèdera à une représentation d'un élément du profil sur lequel porte l'activité qu'il réalise. Par exemple, une représentation textuelle pourrait être disponible à condition que l'utilisateur maîtrise la lecture. De même, une activité de comparaison du profil d'un apprenant avec le profil de classe pourrait être disponible à condition que la moyenne de cet apprenant soit supérieure à 12/20.

Une condition peut porter sur plusieurs critères, c'est pourquoi nous définissons une condition comme une formule logique créée à partir d'une combinaison de contraintes sur les spécificités des apprenants et des utilisateurs. Par exemple, la condition pour qu'une activité ou une représentation soit disponible peut être « $C_{1}$ et $\left(\mathrm{C}_{2}\right.$ ou non $\left.\mathrm{C}_{3}\right)$ », où $\mathrm{C}_{1}, \mathrm{C}_{2}$ et $\mathrm{C}_{3}$ sont des contraintes. Cette condition peut signifier par exemple que l'apprenant maitrise la lecture $\left(\mathrm{C}_{1}\right)$ et que l'utilisateur utilise une synthèse vocale $\left(\mathrm{C}_{2}\right)$ ou bien qu'il n'est pas non-voyant (non $\mathrm{C}_{3}$ ).

Un travail préalable avec des enseignants nous a permis d'établir la nécessité de disposer de trois types de contraintes : des contraintes sur une liste d'apprenants, des contraintes sur le profil d'apprentissage de l'apprenant et des contraintes sur le profil de capacités de l'utilisateur.

Pour les contraintes portant sur un profil, qu'il s'agisse d'un profil d'apprentissage ou de capacités, nous avons choisi d'utiliser le modèle de 
contraintes sur profils cPMDLe (Lefevre, 2009) (Ginon et al., 2011) qui permet de contraindre des profils PMDLe. cPMDLe permet de définir plusieurs types de contraintes: contraintes portant sur une valeur (textuelle ou numérique) ou son évolution, dont la définition est donnée en Figure 6 ; contraintes portant sur un élément ou son évolution et contraintes portant sur un nombre d'occurrences ou son évolution. cPMDLe permet par exemple de sélectionner les apprenants dont la note en Histoire est comprise entre 12 et 14 sur 20, à l'aide d'une contrainte portant sur une valeur numérique, ou de sélectionner les apprenants dont la note en Histoire a augmenté de 1 à 5 points entre deux périodes d'évaluation, à l'aide d'une contrainte portant sur l'évolution d'une valeur numérique. Une contrainte portant sur une valeur textuelle $\mathbf{C l p} \mathbf{p}_{\mathbf{v}(\mathbf{t})}$ concerne un élément Elt, et un ensemble de valeurs val $_{\mathrm{i}}$ qui correspondent aux valeurs permises par la contrainte. Une contrainte portant sur une valeur numérique $\mathbf{C l p}_{\mathbf{v}(\mathbf{n})}$ concerne un élément Elt, ainsi qu'un intervalle défini par les bornes $b_{i}$ et $b_{s}$, $i$ et $s$ étant des booléens qui indiquent si les bornes sont incluses ou non dans l'intervalle ; la note d'un apprenant doit appartenir à cet intervalle pour être concernée par la contrainte. Une contrainte portant sur l'évolution d'une valeur numérique $\operatorname{Clp}_{\mathbf{e v}(\mathbf{n})}$ spécifie également deux dates $\mathrm{d}_{1}$ et $\mathrm{d}_{2}$ qui correspondent à la période dans laquelle on calcule la progression d'un apprenant, qui doit être contenue dans l'intervalle spécifié.

$$
\begin{aligned}
& \operatorname{Clp}_{\mathbf{v}(\mathbf{t})}=\left(\text { Elt, } \operatorname{val}_{1}, \ldots, \mathrm{val}_{2}\right) \\
& \operatorname{Clp}_{\mathbf{v}(\mathbf{n})}=\left(\text { Elt, }\left\{\left\{\mathrm{i}, \mathrm{b}_{\mathrm{i}}\right\},\left\{\mathrm{s}, \mathrm{b}_{\mathrm{s}}\right\}\right\}\right) \\
& \operatorname{Clp}_{\mathbf{e v}(\mathbf{n})}=\left(\text { Elt, }\left\{\left\{\mathrm{d}_{1}, \mathrm{~b}_{1}\right\},\left\{\mathrm{d}_{2}, \mathrm{~b}_{2}\right\}\right\},\left\{\left\{\mathrm{i}, \mathrm{b}_{\mathrm{i}}\right\},\left\{\mathrm{s}, \mathrm{b}_{\mathrm{s}}\right\}\right\}\right)
\end{aligned}
$$

Figure 6. Extrait des modèles de contraintes définis dans cPMDLe

Le modèle cPMDLe permet de définir des contraintes sur les éléments des profils PMDLe, qui peuvent contenir des informations très variées sur l'apprenant. Néanmoins, que faire lorsqu'un enseignant souhaite personnaliser une séance d'activités en fonction d'une information qui ne figure pas dans le profil de l'apprenant? Il est bien sûr possible de modifier la structure des profils pour y ajouter une information absente jugée pertinente par un enseignant. Il est également intéressant de permettre à l'enseignant de personnaliser une séance d'activités en fonction d'informations non explicitées dont lui seul aurait connaissance, afin d'affecter une activité uniquement à certains apprenants par exemple. De plus, même si toutes les informations nécessaires à la personnalisation sont présentes dans le profil des apprenants, certains enseignants peuvent juger plus simple de créer des contraintes de personnalisation pour un sous-ensemble d'un groupe d'apprenants, sans avoir à formaliser les raisons de ce choix de personnalisation par une contrainte sur leur profil, en particulier si les apprenants sont peu nombreux.

Pour cette raison, nous avons étendu cPMDLe avec des contraintes portant sur une liste d'apprenants llist (pour Learner List, cf. Figure 7). Nous définissons une contrainte sur une liste d'apprenants comme une association entre un ensemble d'apprenants $l_{i}$ et un booléen inout indiquant si la condition est une condition d'exclusion $($ inout $=$ false) ou d'inclusion $($ inout $=$ true $)$. Par exemple, la contrainte 
((Julie, Thomas), false) signifie « pour tous les apprenants sauf Julie et Thomas », et la contrainte ((Alice), true) signifie « uniquement pour Alice ».

$$
\text { Cll }(\text { llist })=\left(\left(1_{0}, \ldots, 1_{n}\right), \text { inout }\right)
$$

Figure 7. Modèle de contraintes sur une liste d'apprenants dans cPMDLe

Ces types de contraintes pourront être combinés pour établir des conditions associées à une représentation d'éléments ou à une activité, dans le but de personnaliser une vue sur profils ou une séance d'activités sur les profils en adéquation avec les souhaits des enseignants.

\subsection{Modèle de définition de représentations d'éléments d'un profil : rPMDLe}

S'il existe de nombreuses représentations d'éléments d'un profil, les EIAH existants imposent souvent une ou plusieurs représentations. Certains EIAH permettent néanmoins à l'enseignant de personnaliser des représentations existantes : CosyQTI (Lazarinis et Retalis, 2007) et PépiProfil (Jean, 2000) proposent ainsi aux enseignants de personnaliser une représentation textuelle en modifiant les seuils d'appartenance à une catégorie. Par exemple, l'enseignant peut définir qu'un résultat est considéré comme excellent à partir de $80 \%$ de réussite au lieu de $70 \%$. Toutefois, dans ces systèmes la personnalisation est limitée, puisque l'enseignant ne peut pas modifier le nombre de catégories ni choisir leur nom. Dans UMPTEEN (Bull et al., 2007), les données des profils sont représentées de manière symbolique par des étoiles blanches ou colorées, le nombre d'étoiles colorées par rapport au nombre total d'étoiles représente le niveau de maîtrise de l'apprenant pour un élément. L'utilisateur peut choisir la précision de la représentation en faisant varier le nombre total d'étoiles. Mais, à notre connaissance, aucun EIAH ne propose d'outil permettant à l'enseignant de créer de nouvelles représentations.

Les activités sur les profils d'apprenants sont basées sur la visualisation de profils et en particulier sur la représentation de données contenues dans les profils. Pour permettre une préparation et une personnalisation par l'enseignant de telles activités, il est important de proposer un large choix de représentations d'éléments d'un profil. Néanmoins, il n'est pas possible de prévoir toutes les représentations dont peuvent avoir besoin les enseignants. Les enseignants les plus créatifs, ainsi que ceux ayant des besoins bien particuliers pourraient renoncer à utiliser un outil qui ne leur propose pas les représentations auxquelles ils sont habitués et dont ils ont besoin. C'est pourquoi il est nécessaire de permettre aux enseignants de créer de nouvelles représentations.

Pour cela, nous avons défini rPMDLe, un modèle de représentations d'éléments qui permet la définition de représentations adaptées à tous les éléments de profils respectant le langage de modélisation de profils PMDLe. Pour représenter la valeur associée à un élément d'un profil PMDLe, rPMDLe dispose de trois catégories de représentations (cf. Figure 8) : une représentation adaptée à tous les éléments, des 
représentations adaptées aux éléments associés à une échelle numérique et des représentations adaptées aux éléments associés à une échelle textuelle. Par la suite, elt représente un élément d'un profil et $s$ son échelle respectant sPMDLe. Un astérisque indique qu'un composant est optionnel.

\begin{tabular}{|c|c|c|c|c|}
\hline Échelles & $\begin{array}{l}\text { Textuelle/numérique } \\
\text { 12/20 Acquis }\end{array}$ & Graphique & $\begin{array}{l}\text { Symbolique } \\
\therefore \rightleftharpoons \text { 8 }\end{array}$ & $\begin{array}{l}\text { Audio } \\
\text { (1))) }\end{array}$ \\
\hline Numérique & RnSn & RgSn & RsSn & RaSn \\
\hline Textuelle & RtSt & RgSt & RsSt & RaST \\
\hline Toutes & Rgen & & & \\
\hline
\end{tabular}

Figure 8. Les catégories de représentations proposées par rPMDLe

\subsubsection{Représentations générales d'éléments}

Pour représenter tous les éléments de profils décrits en PMDLe, quel que soit leur type d'échelles, rPMDLe offre une représentation générale, notée Rgen (cf. Figure 8 et Figure 9). Les représentations générales peuvent être utilisées pour associer à un élément d'un profil des instructions de mise en forme pour représenter la valeur de l'élément ou le commentaire associé. $f$ (pour font) représente la police (associée à un nom, un style, une taille et une couleur) et col (pour color) représente la couleur de fond de la représentation. Le booléen disp (pour display) indique si l'échelle associée à l'élément sera ou non affichée ; disp doit valoir false si elt est un commentaire. Les représentations générales peuvent également être utilisées pour indiquer qu'un élément sera lu par une synthèse vocale : dans ce cas, disp indique si l'échelle sera lue avec l'élément, et $f$ et $c o l$ sont absents.

$\operatorname{Rgen}(\mathrm{elt})=\left(\right.$ disp,$\left.\{\mathrm{f}, \mathrm{col}\}^{*}\right)$

Figure 9. Définition de représentations générales d'éléments dans rPMDLe

Pour illustrer ce modèle de représentations générales d'éléments, nous donnons en Figure 10 la définition en rPMDLe de Rgen1, une représentation générale d'éléments qui permet de représenter un élément d'un profil par du texte en Arial noir sur fond blanc, avec une taille de 12 et un style normal : $f=$ (Arial, normal, 12, black) et $c o l=$ white.

Rgen1 $($ elt $)=($ false,$($ Arial, normal, 12, black $)$, white $)$

Figure 10. Exemple d'une représentation générale d'éléments définie en rPMDLe

\subsubsection{Représentations d'éléments associés à une échelle numérique}

Les profils d'apprenants, qu'ils soient issus des pratiques des enseignants ou d'EIAH existants contiennent fréquemment des éléments associés à des échelles numériques, avec des notes sur 10 ou sur 20, des pourcentages ou encore des entiers 
correspondant à un nombre de bonnes réponses. Il est donc essentiel de pouvoir représenter de façon très fine de tels éléments.

Pour représenter un élément d'un profil décrit en PMDLe associé à une échelle numérique, rPMDLe propose quatre types de représentations (cf. Figure 8 et Figure 11): des représentations numériques $(\mathrm{RnSn})$, graphiques (RgSn), symboliques (RsSn) et audio (RaSn). Ces représentations permettent de définir un nombre $k$ d'intervalles numériques de type $\left[\mathrm{a}_{\mathrm{i}}, \mathrm{b}_{\mathrm{i}}\right]$ ou $\left[\mathrm{a}_{\mathrm{i}}, \mathrm{b}_{\mathrm{i}}[\right.$, dont la réunion couvre l'ensemble de l'intervalle de définition [min, max] de l'échelle $s$ associée à elt, avec $\mathrm{a}_{0}=$ min, $\mathrm{b}_{\mathrm{k}}=\max , \mathrm{b}_{\mathrm{i}}=\mathrm{a}_{\mathrm{i}+1}, \mathrm{a}_{\mathrm{i}}<\mathrm{b}_{\mathrm{i}}$ et $0<\mathrm{i}<=\mathrm{k}$.

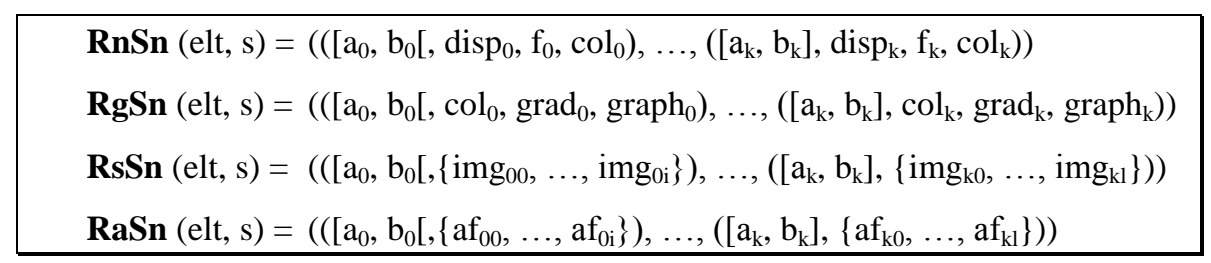

Figure 11. Définition de représentations d'éléments associés à une échelle numérique dans rPMDLe

Une représentation numérique RnSn permet de définir une mise en forme pour chaque intervalle numérique. Les enseignants utilisent souvent des représentations numériques, parfois en les associant à un code couleur pour écrire la note d'un apprenant en rouge ou en vert selon qu'elle est bonne ou mauvaise par exemple. De telles représentations peuvent être définies à l'aide du modèle de représentation numérique d'éléments d'échelle numérique RnSn.

Parmi les EIAH proposant une visualisation de profils, les éléments associés à une échelle numérique sont souvent représentés de manière graphique. C'est le cas par exemple dans SQL-Tutor (Mitrovic et Martin, 2007) qui représente les pourcentages par des barres de progression dont la couleur peut varier en fonction du pourcentage représenté. Pour définir de telles représentations, le modèle de représentations graphiques $\mathrm{RgSn}$ permet d'associer à chaque intervalle une couleur $\mathrm{col}$, un booléen grad (pour gradation) indiquant si la couleur sera enrichie d'un dégradé ou non, et un objet graphique graph (barre de progression, thermomètre, compteur...).

Les éléments associés à une échelle numérique peuvent être représentés de manière graphique. On retrouve de telles représentations dans les pratiques enseignantes, mais aussi dans certains EIAH, qu'ils soient à destination d'apprenants adultes, comme c'est le cas pour UMPTEEN (Bull et al., 2007), ou à destination de jeunes apprenants, comme c'est le cas dans Fractionator (Bull et al., 2005). Pour définir de telles représentations, notre modèle de représentations symboliques d'éléments d'échelle numérique RsSn permet d'associer à chaque intervalle une ou plusieurs images Img (smiley, flèche, médaille...) associées aux caractéristiques suivantes : nombre d'occurrences, taille et couleur (pouvant être la couleur par 
défaut de l'image: def). La Figure 19 (cf. section 4.2) donne l'exemple d'une représentation symbolique utilisée dans UMPTEEN et écrite en rPMDLe.

Dans certains cas, les éléments dont l'échelle est numérique peuvent être associés à une représentation audio, notamment pour les personnes malentendantes et les apprenants ne maîtrisant pas la lecture. Pour définir de telles représentations, notre modèle de représentation audio d'éléments d'échelle numérique RaSn permet d'associer à chaque intervalle un ou plusieurs fichiers audio af (pour audio file), associés à un nombre de répétitions et à un volume sonore.

\subsubsection{Représentations d'éléments associés à une échelle textuelle}

Les profils d'apprenants, qu'ils soient issus des pratiques des enseignants ou des EIAH existants contiennent parfois des éléments associés à des échelles textuelles, comme «maîtrisé/ partiellement maîtrisé/ non maîtrisé », ou « acquis/ non acquis ». Il est donc nécessaire de permettre la représentation de tels éléments.

Pour représenter un élément d'un profil PMDLe associé à une échelle textuelle, rPMDLe propose quatre types de représentations (cf. Figure 8 et Figure 12), qui sont le pendant de celles pour des éléments associés à une échelle numérique. Avec les éléments d'échelle numérique, une représentation concerne des intervalles numériques, alors qu'avec les éléments d'échelle textuelle, une représentation concerne les $n$ niveaux lev (pour Level) de l'échelle. De plus, pour représenter graphiquement un niveau d'une échelle textuelle, il est nécessaire de spécifier un entier int correspondant à ce niveau, ce qui équivaut à convertir l'échelle textuelle en une échelle numérique.

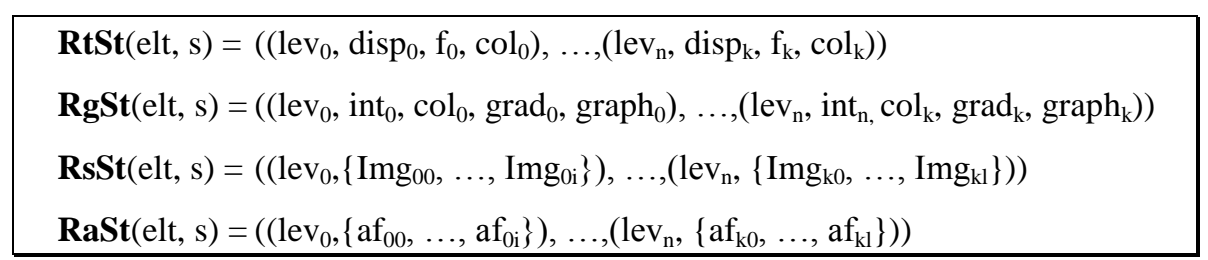

Figure 12. Définition de représentations d'éléments associés à une échelle textuelle dans $r$ PMDLe

\subsubsection{Représentations graphiques composées d'éléments}

Les EIAH proposant une visualisation de profils proposent parfois de visualiser plusieurs valeurs sur une même représentation. Par exemple, dans MoreMaths (Bull et al., 2003), les éléments contenus dans les profils sont associés à trois composantes («corrects answers », «wrong answers », « unanswered »), chacune valuée par un nombre entier. Par ailleurs, une représentation contenant les valeurs provenant de deux profils d'apprenants permet de les comparer, afin de situer un apprenant par rapport à sa classe par exemple, comme c'est le cas dans CosyQTI (Lazarinis et Retalis, 2007). Une représentation composée de plusieurs éléments peut également permettre de représenter la répartition des notes d'un groupe 
d'apprenants, avec un histogramme, ou l'évolution d'un apprenant, avec une courbe par exemple. Pour définir de telles représentations, nous proposons dans rPMDLe une catégorie de représentations graphiques composées, notée Rcomp (cf. Figure 13) qui permet de représenter plusieurs éléments ou plusieurs valeurs d'un ou plusieurs éléments sur un même graphique.

Dans la définition des représentations graphiques composées, type est un type de représentations graphiques composées (diagramme en barres, en étoiles... cf. Figure 14), dim est un entier représentant le nombre de dimensions (2 ou 3), 3Deff (pour 3D effect) est un booléen indiquant si un diagramme en 2D a un effet de relief, bar représente le type de barres (pavés ou cylindres) et orient indique si les barres seront horizontales ou verticales. De plus, tit $_{i}$ (pour title) est une chaîne de caractères spécifiant le nom du sous-titre $\mathrm{i}$; $\mathrm{col}_{j}$ est la couleur de la série $\mathrm{j}$, rbgcol (pour representation background color) est la couleur de fond de la représentation, gbgcol (pour graphic background color) est la couleur d'arrière-plan du graphique, gcol (pour grid color) est la couleur du quadrillage, pref est un booléen indiquant si les préférences de l'utilisateur seront prises en compte pour les couleurs et polices, $f_{i}$ est la police du sous-titre $i$.

$\operatorname{Rcomp}(\mathrm{elt}, \mathrm{s})=\left(\right.$ type $, \operatorname{dim}^{*}, 3 \operatorname{Deff} *$, bar*, orient*, $\left(\mathrm{tit}_{0}, \ldots, \mathrm{tit}_{\mathrm{k}}\right)^{*},\left(\mathrm{col}_{0}, \ldots, \mathrm{col}_{1}\right)$, rbgcol, cbgcol, gcol*, pref, $\left.\left.\left(\mathrm{f}_{0}, \ldots, \mathrm{f}_{\mathrm{m}}\right)^{*}, \mathrm{sf}^{*},\left\{\operatorname{dev}_{\mathrm{k} 0}, \ldots, \operatorname{dev}_{\mathrm{km}}\right\}^{*}\right)\right)$

Figure 13. Définition de représentations d'éléments non terminaux dans rPMDLe

La Figure 14 illustre par quelques exemples des représentations graphiques composées que rPMDLe permet de définir.

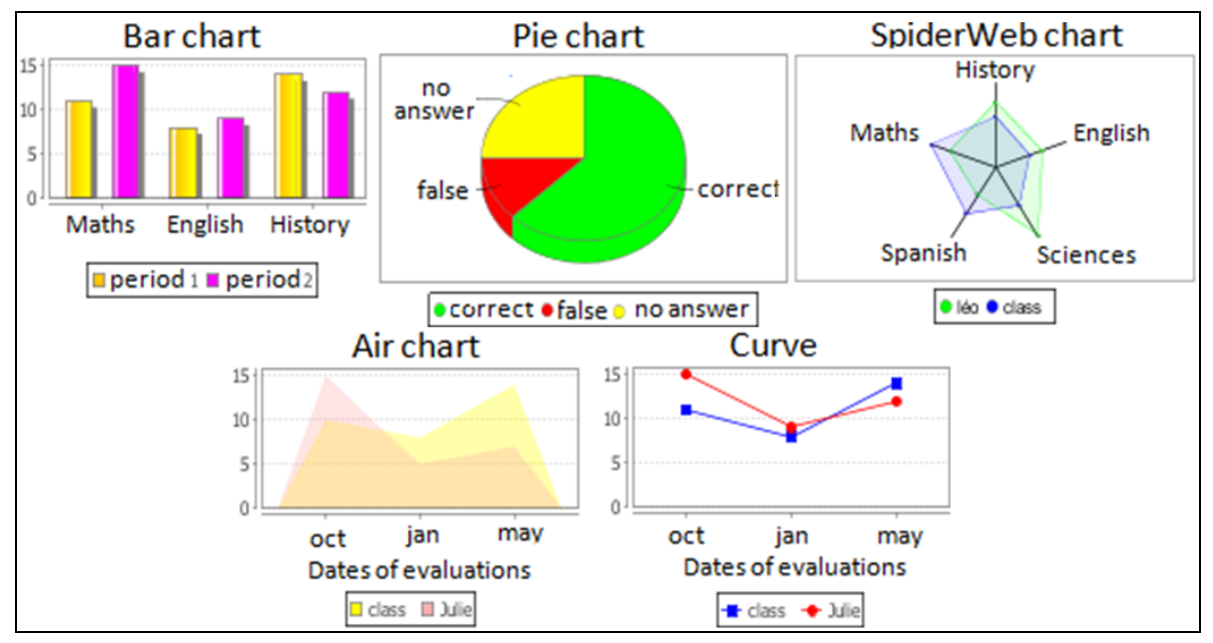

Figure 14. Exemples de représentations graphiques composées 


\subsection{Modèle de vues sur profils : vPMDLe}

Afin de proposer à chaque utilisateur une visualisation personnalisée d'un profil, il est nécessaire de déterminer quels éléments du profil seront visualisables par quels utilisateurs et de quelles manières.

Certains EIAH qui ouvrent les profils qu'ils maintiennent à leurs utilisateurs ne présentent pas la totalité du contenu du profil. Ceci s'explique par le trop vaste contenu du profil, qui surchargerait d'informations l'utilisateur, ou par la volonté de masquer certaines informations utilisées par l'EIAH mais non destinées aux apprenants. Néanmoins, l'enseignant n'a dans ces systèmes aucune possibilité de contrôle sur la sélection des éléments du profil qui seront visibles pour les utilisateurs. Par ailleurs, quelques EIAH proposent une visualisation adaptée à différents types d'utilisateurs, comme Substraction Master, un EIAH destiné à des enfants de 8 à 9 ans (Bull et McKay, 2004). Il propose d'une part aux apprenants une visualisation du contenu de leur profil sous forme symbolique à l'aide de smileys, et d'autre part aux enseignants une visualisation du contenu des profils des apprenants sous forme graphique et textuelle. Toutefois, le choix des représentations pour les utilisateurs est fait par le système, l'enseignant n'a pas la possibilité de choisir lui-même quelles représentations seront proposées à un type d'utilisateurs. En outre, l'enseignant peut souhaiter attribuer des représentations différentes à deux utilisateurs d'un même type. Par exemple, un enseignant peut vouloir représenter les éléments d'un profil de manière symbolique pour tous les apprenants, et ajouter une représentation textuelle précisant le niveau de maîtrise de l'apprenant uniquement pour les apprenants sachant lire. Cette personnalisation en fonction des capacités et connaissances de l'apprenant n'est pas proposée par les travaux existants sur la visualisation. Il existe par contre des travaux sur la personnalisation de l'apprentissage qui permettent de prendre en compte les capacités et connaissances d'un apprenant. Ces travaux sont basés sur la définition de règles sous la forme « if $X$ then $Y$ else $Z$ ». Par exemple CosyQTI (Lazarinis et Retalis, 2007) et aLFanet (Van Rosmalen et al., 2006) permettent à l'enseignant de définir des règles pour personnaliser l'enchaînement des questions dans des QCM ou des questionnaires d'auto-évaluation. Adapte (Lefevre, 2009) permet quant à lui de personnaliser des séances d'utilisation d'un EIAH ou des feuilles d'exercices à l'aide de règles. De plus, dans Flexi-OLM (Bull et Mabbott, 2006b) et OLMlet (Bull et Mabbott, 2006a) il existe plusieurs représentations parmi lesquelles l'apprenant peut choisir. Néanmoins, l'enseignant ne peut pas imposer ou interdire l'utilisation d'une représentation.

Pour permettre de définir quels éléments d'un profil décrit en PMDLe seront visibles au cours d'une activité sur le profil d'un apprenant en fonction du type d'utilisateurs, des connaissances de l'apprenant et des capacités de l'utilisateur, nous proposons le modèle de vues sur profils vPMDLe (cf. Figure 5). vPMDLe permet de définir des vues (cf. Figure 15), pour une structure de profils d'apprentissage $s t r L P$, une structure de profils de capacités $\operatorname{str} C P$, un type d'utilisateurs userT, une liste 
d'apprenants llist et une catégorie d'activités sur les profils actT. Une vue permet d'associer à chaque élément elt d'un profil zéro, une ou plusieurs représentations $R e p$ respectant rPMDLe, elles-mêmes pouvant être associées à une condition $C$. Une représentation ne sera disponible que si la condition $\mathrm{C}$ qui lui est associée est vérifiée, $\mathrm{C}$ étant une formule logique combinant des contraintes définies en cPMDLe. Il est également possible d'attribuer à un élément un intitulé head (pour heading), afin que cet élément soit désigné par cet intitulé lors de l'activité. Par exemple, un enseignant en école primaire peut souhaiter présenter l'élément «Exercices algébriques » avec l'intitulé « Problèmes», plus compréhensible pour de jeunes écoliers.

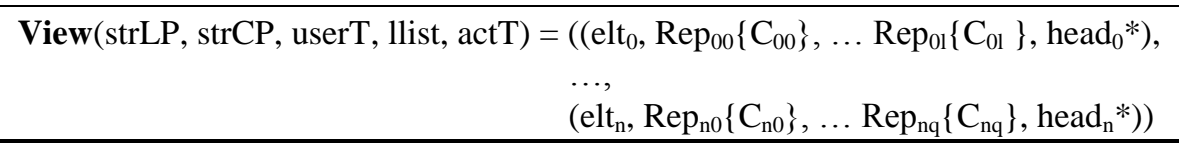

Figure 15. Définition formelle de vues sur profils selon le modèle vPMDLe

La Figure 20 (cf. section 4.2) donne l'exemple d'une vue sur profils en vPMDLe équivalente à celle utilisée par UMPTEEN.

\subsection{Définition de séances d'activités sur les profils}

Afin de proposer à chaque utilisateur des activités personnalisées sur les profils, l'enseignant doit pouvoir déterminer quelles activités seront accessibles pour un utilisateur, mais aussi de quelle manière sera représenté le profil d'apprenant dans cette activité.

Les EIAH offrant une ou plusieurs activités sur les profils ne permettent pas à l'enseignant de contrôler l'accès à ces activités, comme dans Mr. Collins (Bull et Pain, 1995). Toutefois, certains logiciels utilisés par les établissements scolaires pour le suivi des élèves permettent de masquer certaines informations en fonction du type d'utilisateurs, comme c'est le cas dans Nota Bene (Nota_Bene, 2010). Il peut également être pertinent de ne pas montrer les mêmes éléments à deux utilisateurs du même type. Ainsi, un enseignant peut choisir de masquer certains résultats pour un apprenant très faible, afin d'insister plutôt sur les points positifs de son profil pour ne pas le décourager. À notre connaissance, cette personnalisation n'est pas possible avec les systèmes actuels.

Session(strLP, $\operatorname{strCP}, \operatorname{strPP}$, userT, llist $)=\left(\mathrm{Act}_{0}\left\{\mathrm{View}_{0}, \mathrm{C}_{0}\right\}, \ldots \mathrm{Act}_{\mathrm{n}}\left\{\mathrm{View}_{\mathrm{n}}, \mathrm{C}_{\mathrm{n}}\right\}\right)$

Figure 16. Définition de séances d'activités sur les profils

Pour permettre de déterminer quelles activités sur les profils seront disponibles pour quels utilisateurs, nous avons défini la notion de séance d'activités (cf. Figure 16). Une séance d'activités concerne une structure de profils d'apprentissage $s t r L P$, une structure de profils de capacités $s t r C P$, une structure de profils de préférences 
strPP (toutes trois écrites en PMDLe), un type d'utilisateurs userT et une liste d'apprenants llist. Une séance d'activités est composée d'un ensemble d'activités $A c t$, chacune associée à une vue View et à une condition $C$. Une activité $A c t_{i}$ sera disponible pour un utilisateur s'il vérifie la condition $C_{i}$ associée à l'activité, ou si cette condition est vide. Au cours d'une activité $A c t_{i}$ le profil concerné sera représenté grâce à la vue $\operatorname{View}_{i}$.

La Figure 21 (cf. section 4.2) donne l'exemple d'une séance d'activités équivalente à celle proposée dans UMPTEEN.

\section{4. Évaluation des modèles proposés}

Nous avons associé des critères de validation à chacune des questions de recherche posées par ce travail sur les activités de visualisation de profils.

Est-il possible de proposer, tout en adoptant une démarche générique, un environnement informatique destiné aux enseignants afin de leur permettre de concevoir et de personnaliser des activités sur les profils d'apprenants ? La faisabilité de notre approche est prouvée par la mise en œuvre dans l'environnement EPROFILEA des modèles présentés dans cet article (cf. section 2.4).

Dans quelle mesure un système s'appuyant sur des modèles génériques peut-il proposer des activités sur les profils aussi riches que les systèmes ad hoc existants ? Nous cherchons en particulier à savoir si un modèle générique de représentations d'éléments peut permettre la définition des représentations employées dans les pratiques des enseignants et les travaux issus des recherches en EIAH. Nous évaluons ce point en tentant de reproduire les activités existantes à l'aide de nos outils afin d'en tester la couverture (cf. section 4.1).

Est-il possible d'exploiter des modèles génériques dans un contexte autre que celui dans lequel ils ont été mis en œuvre ? Nous évaluons ce point en montrant qu'il est possible de réutiliser de plusieurs manières nos modèles, initialement conçus pour exploiter des profils PMDLe, dans le cadre d'EIAH utilisant des profils respectant un formalisme autre que PMDLe (cf. section 4.2 pour un exemple).

Les activités sur les profils que nous proposons, ainsi que leur personnalisation, répondent-elles aux besoins des acteurs de l'apprentissage ? Un tel système, mettant en œuvre des modèles génériques, sera-t-il utilisable par des enseignants ? Pourra-til s'intégrer dans leurs pratiques ? Afin d'évaluer ces différents aspects, nous avons réalisé une étude des pratiques des enseignants et de l'existant, complétée par plusieurs mises à l'essai de nos outils (cf. section 4.3).

\section{1. Étude de la couverture des modèles}

Afin d'évaluer la couverture de rPMDLe, notre modèle de représentations d'éléments, et de vPMDLe, notre modèle de vue sur profils, nous avons étudié l'existant en matière de visualisation des profils, d'une part dans les pratiques des 
enseignants et d'autre part dans les recherches en EIAH, notamment dans les travaux concernant le modèle ouvert de l'apprenant (McCalla et al., 2007).

\begin{tabular}{|c|c|c|c|}
\hline rPMDLe & EIAH & $\begin{array}{l}\text { Type de } \\
\text { représentation }\end{array}$ & Aperçu \\
\hline$\checkmark$ Oui & Mr. Collins & textuelle & - almost sure (b) \\
\hline$\checkmark$ Oui & SQL-Tutor & $\begin{array}{l}\text { Graphique } \\
\text { Numérique/textuelle }\end{array}$ & $33 \%$ covered $25 \%$ learned \\
\hline J Oui & e-Kermit & $\begin{array}{l}\text { Graphique } \\
\text { Numérique/textuelle }\end{array}$ & $33 \%(44 \%)$ \\
\hline S Oui & C-Polmile & $\begin{array}{l}\text { Graphique } \\
\text { Numérique/textuelle }\end{array}$ & \begin{tabular}{l|l|}
50 & 25
\end{tabular} \\
\hline$\checkmark$ Oui & MoreMaths & $\begin{array}{l}\text { Graphique composée } \\
\text { Numérique/textuelle }\end{array}$ & $\begin{array}{l}\text { You seem to partially understand the concept } \\
\text { of degrees of polynomials }\end{array}$ \\
\hline$\checkmark$ Oui & $\begin{array}{l}\text { Substraction } \\
\text { Master }\end{array}$ & $\begin{array}{l}\text { Graphique composée } \\
\text { Symbolique }\end{array}$ & 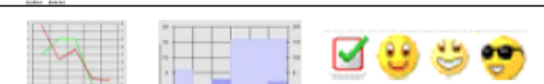 \\
\hline$\checkmark$ Oui & Sam Text editor & Symbolique & 口scrol113_k \\
\hline$\checkmark$ Oui & UMPTEEN & $\begin{array}{l}\text { Symbolique } \\
\text { Textuelle }\end{array}$ & . you may believe that while loop and do while loop are equivalent \\
\hline J Oui & CosyQTI & $\begin{array}{l}\text { Graphique composée } \\
\text { Numérique/textuelle }\end{array}$ & $80.0 \%$ (Vely good) \\
\hline$\checkmark$ Oui & Flexi-OLM & Symbolique & $\square$ If construct $\square$ printf $\square$ Arrays $\square$ Prefixes \\
\hline S Oui & OLM-lets & $\begin{array}{l}\text { Graphique } \\
\text { Graphique composée }\end{array}$ & \\
\hline J Oui & Wandies & Symbolique & \\
\hline$\checkmark$ Oui & QuizPack & Symbolique & \\
\hline$\checkmark$ Oui & i-Start-me & $\begin{array}{l}\text { Graphique } \\
\text { Numérique/textuelle }\end{array}$ & Serious Strategizer \\
\hline$\checkmark$ Oui & ERR-Tutor & $\begin{array}{l}\text { Graphique } \\
\text { Numérique/textuelle }\end{array}$ & ed: $\mathbf{7 9} \%$, learned: $\mathbf{7 0} \%$ \\
\hline$\times$ Non & STyLE-OLM & Graphique & \\
\hline$\times$ Non & Introspective View & Graphique & \\
\hline$\times$ Non & VisMod & Graphique & \\
\hline$\times$ Non & Flexi-OLM & Graphique & 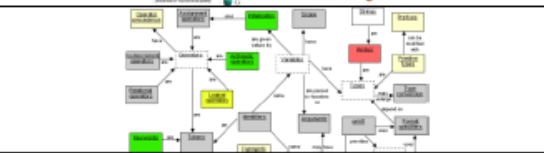 \\
\hline
\end{tabular}

Figure 17. Couverture de rPMDLe : définition de représentations identiques à celles utilisées dans des EIAH variés 
Nous avons également étendu notre étude aux logiciels du commerce et aux logiciels qui sortent du cadre des EIAH, notamment aux jeux vidéo. En effet, dans ce domaine il existe de nombreuses représentations de scores de joueurs, pouvant s'apparenter à des profils d'apprenants. Les résultats généraux de notre étude sont résumés dans la suite de cette section. En exploitant les modèles génériques présentés dans cet article, nous détaillerons à titre d'exemple dans la section suivante, comment il est possible d'obtenir une visualisation de profil similaire à celle utilisée dans UMPTEEN.

Cette étude nous a permis de vérifier que rPMDLe rend possible la définition formelle de toutes les représentations issues des pratiques des enseignants que nous avons pu observer. Il permet également de représenter la plupart des représentations utilisées dans les EIAH que nous avons étudiés. La Figure 17 présente un extrait des représentations issues des EIAH que nous avons pu reproduire avec rPMDLe, ainsi que les représentations que rPMDLe ne permet pas de définir. Ainsi, rPMDLe ne permet pas de reproduire la représentation d'Introspective View, une représentation graphique très particulière et que l'on ne trouve que dans cet EIAH. Pour les autres représentations non reproductibles par rPMDLe, c'est la nature de PMDLe qui est en cause. Tout d'abord, PMDLe ne permet pas de représenter pleinement les réseaux bayésiens (Ginon et Jean-Daubias, 2012). En ce qui concerne la représentation sous forme de graphe proposée dans Flexi-OLM, nos outils ne permettent pas non plus de la modéliser. Dans ce graphe, les regroupements d'éléments se font a posteriori par valeur, en opposition à la vision classique des profils dans laquelle les regroupements sont faits a priori par thématique.

\subsection{Réutilisabilité des modèles}

Les différents modèles présentés dans cet article complètent le langage de modélisation de profils PMDLe. Ils s'appuient sur sa généricité qui permet la définition de profils très variés, dans différents domaines et à différents niveaux. La réutilisation de nos modèles peut se faire selon deux approches : soit seuls, soit via leur mise en œuvre. En effet, un EIAH peut déléguer à l'environnement EPROFILEA la gestion des activités sur les profils, ou le modèle PERSUMAP peut être appliqué indépendamment de PMDLe dans un EIAH utilisant un autre formalisme pour la gestion des profils d'apprenants.

Tout d'abord, nos modèles peuvent permettre de personnaliser des séances d'activités sur des profils issus de différents EIAH ou des pratiques des enseignants. Ainsi, il est possible de déléguer à l'environnement EPROFILEA la gestion des profils issus d'un logiciel externe et celle des activités sur ces profils, afin d'apporter un complément à ce logiciel. En effet, à l'aide des modules Tornade et Prose (JeanDaubias et al., 2009) d'EPROFILEA, il est possible d'intégrer des données issues de logiciels externes dans des profils respectant PMDLe, et donc exploitables par tous les modules d'EPROFILEA. En particulier, les profils ainsi intégrés pourront faire 
l'objet de séances d'activités sur les profils, préparées et personnalisées par l'enseignant à l'aide du module Regards, et effectuées par tous les utilisateurs à l'aide du module Perl.

Par ailleurs, les modèles présentés dans cet article peuvent être utilisés indépendamment de PMDLe et d'EPROFILEA, par exemple dans le cadre d'un EIAH utilisant un formalisme autre que PMDLe ou une structure de profils fixe. Dans ce cas, les modèles que nous avons proposés seront sous-exploités puisque les contraintes, les représentations d'éléments et les vues sur profils qu'ils permettront de définir dans ce contexte seront uniquement adaptés à la structure fixe et aux éléments des profils proposés par cet EIAH. De plus, les séances d'activités sur les profils ne concerneront dans ce contexte que la personnalisation de la ou des activités proposées par cet EIAH et non plus la personnalisation de toutes les activités que nous avons identifiées dans notre typologie des activités sur les profils d'apprenants et que nous avons implémentées dans Perl. Cette réutilisation est toutefois possible et peut simplifier le travail des concepteurs d'EIAH. Afin d'illustrer cette réutilisabilité de nos modèles indépendamment de PMDLe, nous développons dans la suite de cette section l'exploitation de ces modèles pour l'EIAH UMPTEEN (Bull et al., 2007).

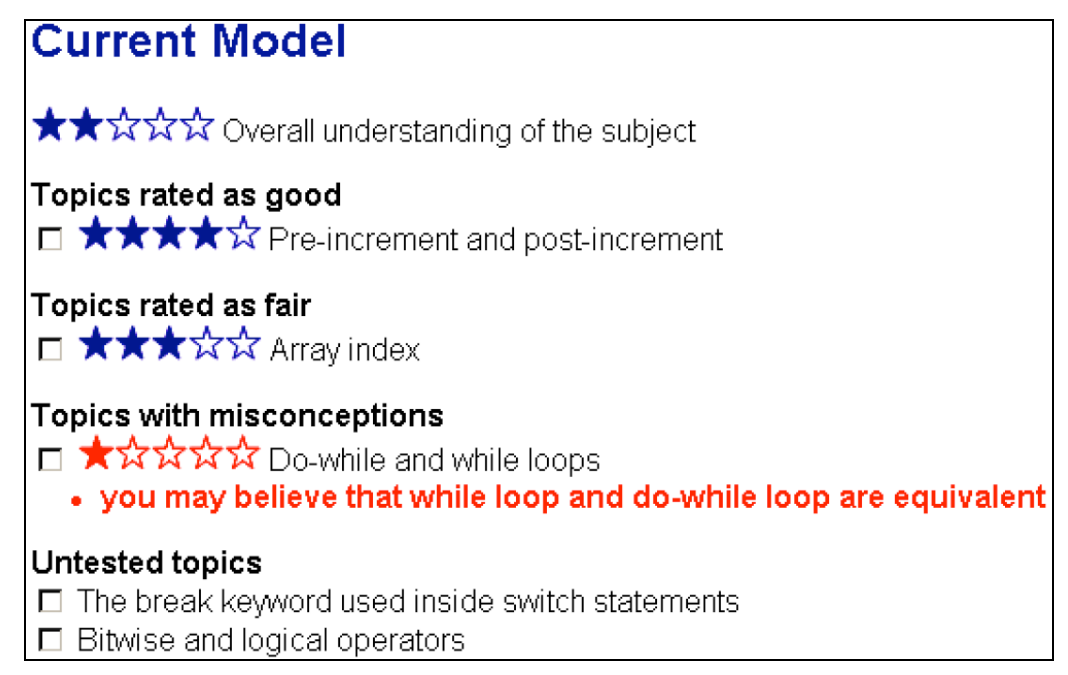

Figure 18. Profil d'un apprenant utilisé dans UMPTEEN

UMPTEEN est un système de visualisation de profils d'apprenants indépendant du domaine de connaissances (Bull et al., 2007). Dans UMPTEEN, un enseignant peut créer une structure de profils simple, avec une généricité limitée : il peut créer autant d'éléments qu'il le souhaite, mais ces éléments ne peuvent pas avoir de lien hiérarchique entre eux. La Figure 18 présente un profil d'apprenant utilisé dans 
UMPTEEN : ce profil contient cinq éléments qui sont obligatoirement valués par un nombre entre 0 et 1 correspondant au taux de réussite de l'apprenant au QCM défini par l'enseignant, relativement à l'élément. Si l'enseignant le souhaite, certains éléments du profil peuvent être associés à d'autres valeurs qui correspondent aux conceptions erronées de l'apprenant. Pour cela, l'enseignant a identifié ces conceptions erronées parmi les mauvaises réponses possibles d'un QCM, et la valeur associée à une conception erronée correspond à la probabilité que l'apprenant ait cette conception erronée.

Intéressons-nous maintenant à la manière d'utiliser les modèles que nous avons présentés dans cet article sur des profils respectant le formalisme de UMPTEEN et non pas PMDLe. Concernant la définition de contraintes à l'aide de cPMDLe, les contraintes porteront sur un score ou une conception erronée associé à un élément d'un profil défini dans UMPTEEN, les contraintes seront uniquement de type numérique et associées à l'échelle «nombre entre 0 et 1 ». Concernant la définition de représentations d'éléments d'un profil avec rPMDLe, là encore seules des représentations adaptées à l'échelle numérique «nombre entre 0 et 1 » pourront être définies et utilisées. L'utilisation de rPMDLe dans UMPTEEN permettrait de définir des représentations très variées adaptées à cette échelle, y compris la représentation proposée dans UMPTEEN (cf. Figure 18), qui est composée d'étoiles pleines et d'étoiles vides dont la définition en rPMDLe est donnée en Figure 19.

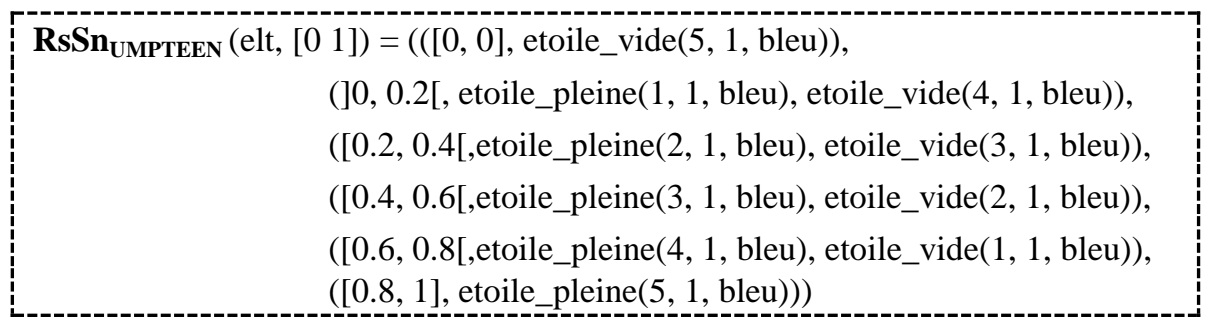

Figure 19. Définition en rPMDLe d'une représentation utilisée dans UMPTEEN

Dans le contexte d'UMPTEEN, une vue sur profil décrite avec vPMDLe permettrait de définir la manière dont un profil UMPTEEN sera représenté dans une activité de la même manière que pour les profils PMDLe : un élément d'un profil défini dans UMPTEEN pourra être associé à zéro, une ou plusieurs représentations, elles-mêmes éventuellement associées à une condition sous forme de formule logique combinant des contraintes cPMDLe. La définition en vPMDLe d'une vue sur profils équivalente à la manière dont les profils sont visualisés dans UMPTEEN (cf. Figure 18) est donnée à titre d'exemple sur la Figure 20. 


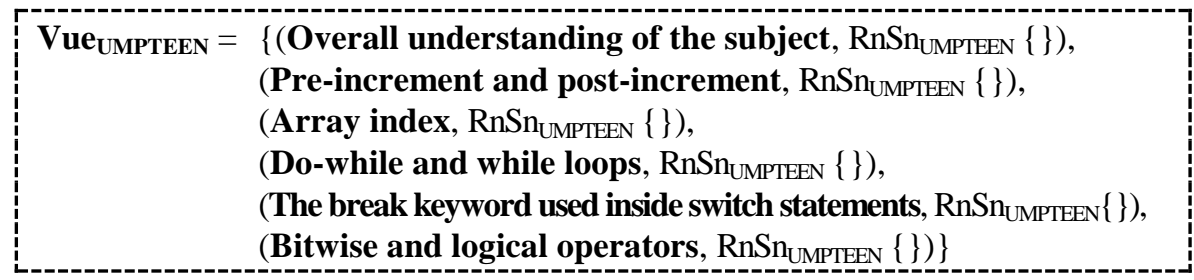

Figure 20. Définition en vPMDLe d'une vue adaptée à un profil d'UMPTEEN

Enfin, les séances d'activités sur les profils définis dans le contexte d'UMPTEEN ne pourront porter que sur les activités proposées par cet EIAH : visualiser son profil, visualiser le profil d'un pair et visualiser la cartographie du groupe. Une activité pourra être associée à une vue sur profil vPMDLe et éventuellement à une condition spécifiant dans quels cas un apprenant pourra accéder à cette activité, de la même manière que pour des séances d'activités portant sur des profils respectant PMDLe. La définition de la séance d'activités proposée dans UMPTEEN est donnée à titre d'exemple sur la Figure 21.

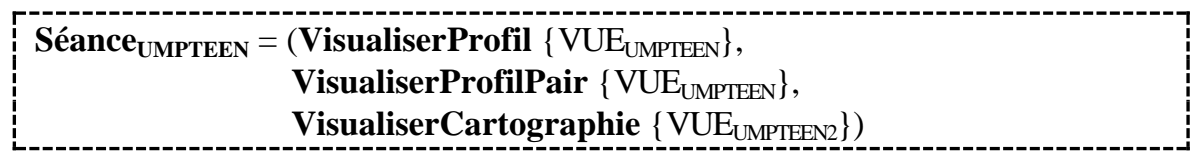

Figure 21. Définition de la séance d'activités proposée dans UMPTEEN

Les modèles que nous proposons peuvent donc être réutilisés indépendamment de PMDLe et d'EPROFILEA. Ils permettent ainsi de modéliser et éventuellement d'enrichir et de personnaliser les activités sur les profils d'apprenants proposées par un EIAH utilisant un autre formalisme que PMDLe.

\subsection{Mise à l'essai des systèmes mettant en auvre les modèles}

Nous avons organisé plusieurs mises à l'essai des modules Regards et Perl, afin de tester l'utilisabilité et l'utilité de notre dispositif.

La première mise à l'essai impliquait 48 étudiants de Master en situation d'apprentissage dans l'un de leurs cours. Nous leur avons demandé d'effectuer dans Perl des activités sur leurs profils. En tant qu'enseignantes, nous avions préalablement préparé à l'aide de Regards une séance d'activités personnalisée à leur intention. Une séance d'activités durait environ 10 minutes et était suivie par un questionnaire de satisfaction. Les apprenants ont réalisé sans problème les trois activités qui leur étaient proposées : visualisation de leur profil, visualisation de l'évolution de leur profil et comparaison de leur profil avec le profil du groupe. Ils ont déclaré avoir compris le contenu de leur profil, et l'intérêt des activités qui leur ont été proposées. Ils ont également apprécié la diversité et la richesse des 
représentations d'éléments proposées dans Perl. Néanmoins, cette mise à l'essai a mis en évidence que le design de Perl nécessite d'être amélioré afin d'augmenter son attractivité.

La deuxième mise à l'essai de notre approche, plus globale, a été réalisée cette fois du point de vue des enseignants. 66 étudiants de Master informatique ont joué le rôle d'enseignants et ont utilisé l'environnement EPROFILEA dans sa totalité. Ils ont notamment utilisé le module Regards pour créer des séances d'activités personnalisées, ainsi que des contraintes sur profils, des représentations d'éléments, et des vues sur profils. Les étudiants ont ensuite joué le rôle d'apprenants aux connaissances très variés afin de tester dans Perl les séances d'activités personnalisées qu'ils avaient préparées avec Regards. Cette étude a permis de confirmer la richesse des possibilités de personnalisation offertes par les modèles et les outils que nous proposons.

Afin de tester l'utilisabilité de Regards par des enseignants non informaticiens, nous avons réalisé une mise à l'essai avec une enseignante de secrétariat en lycée professionnel. Nous lui avons demandé d'utiliser Regards afin de définir une activité de visualisation de profils. Les profils contenaient les résultats de 5 épreuves, chacune composée de 12 à 15 éléments associés à deux valeurs (numérique et textuelle). La préparation de cette activité de visualisation a demandé 1 h30 à cette enseignante, novice en informatique, mais ayant reçu une explication de l'utilisation de Regards. Le plus long a été la création de cinq représentations d'éléments qui pourront être réutilisées par cette enseignante ou ses collègues pour de prochaines activités sur les profils. Nous avons ensuite demandé à un autre enseignant de visualiser dans Perl huit profils d'élèves choisis au hasard parmi ceux issus du travail précédent. L'enseignant a trouvé la visualisation claire et agréable, l'aidant à comprendre les résultats des élèves plus facilement que dans le fichier Excel habituel.

\subsection{Synthèse des évaluations réalisées}

Les différentes évaluations que nous avons réalisées nous ont permis de valider entièrement ou partiellement plusieurs critères de validation, cf. Figure 22. Revenons sur les critères validés seulement partiellement. L'étude de la couverture des modèles rPMDLe et vPMDLe a montré que la plupart des représentations et des vues sur profils peuvent être représentés à l'aide de nos modèles. Néanmoins, rPMDLe ne permet pas la définition de certaines représentations graphiques spécifiques. Par ailleurs, les mises à l'essai de nos outils ont permis de montrer l'utilité de Regards et de Perl pour les enseignants comme pour les apprenants. Cependant, elles ont également mis en évidence des limites du point de vue de 
l'utilisabilité : des améliorations concernant l'ergonomie et le design doivent être réalisées afin d'améliorer l'attractivité de Perl.

\begin{tabular}{|c|c|c|}
\hline Critère & Travail réalisé pour évaluer ce critère & Résultat \\
\hline $\begin{array}{l}\text { Couverture } \\
\text { des modèles }\end{array}$ & $\begin{array}{l}\text { - Étude des pratiques des enseignants } \\
\text { - Étude des profils existants } \\
\text { - Utilisation de rPMDLe et vPMDLe pour imiter les } \\
\text { représentations et les vues sur profils existantes }\end{array}$ & $\sim$ \\
\hline $\begin{array}{l}\text { Réutilisabilité } \\
\text { des modèles }\end{array}$ & $\begin{array}{l}\text { - Possibilité de convertir des profils issus d'EIAH } \\
\text { n'utilisant pas PMDLe } \\
\text { - Possibilité de réutiliser les modèles proposés } \\
\text { indépendamment de PMDLe et d'EPROFILEA }\end{array}$ & $\sqrt{ }$ \\
\hline $\begin{array}{l}\text { Faisabilité } \\
\text { de l'approche }\end{array}$ & - Implémentation complète des modèles proposés & $\sqrt{ }$ \\
\hline $\begin{array}{l}\text { Utilité des outils } \\
\text { et de l'approche }\end{array}$ & $\begin{array}{l}\text { - Étude des pratiques des enseignants et de } \\
\text { l'existant } \\
\text { - Questionnaire de satisfaction pour des apprenants } \\
\text { suite à une utilisation de Perl } \\
\text { - Retours d'enseignants suite à l'utilisation de } \\
\text { Regards et Perl }\end{array}$ & $\sqrt{ }$ \\
\hline Utilisabilité des outils & $\begin{array}{l}\text { - Mises à l'essai de Regards par des enseignants et } \\
\text { de Perl par des apprenants }\end{array}$ & $\sim$ \\
\hline
\end{tabular}

Figure 22. Synthèse des évaluations de l'approche et des modèles proposés

\section{Conclusion et perspectives}

Dans le domaine des EIAH, les profils d'apprenants et la personnalisation de l'apprentissage sont au cœur des problématiques actuelles. Les travaux qui font l'objet de cet article combinent ces deux aspects : le modèle de personnalisation multi-aspects des activités sur les profils d'apprenants permet de proposer à chaque utilisateur des activités entièrement personnalisées sur les profils d'apprenants, en fonction des connaissances et compétences des apprenants, mais aussi en fonction des capacités et préférences des utilisateurs. Nous parlons d'activités « entièrement personnalisées » dans le sens où la personnalisation peut concerner tous les aspects d'une séance d'activités : l'accès à une activité, l'accès à un élément du profil et la manière dont cet élément est représenté.

À travers des modèles théoriques, mis en œuvre de façon opérationnelle sous forme d'outils unifiés, nos travaux répondent à la problématique du projet PERLEA. Nous exploitons en effet un langage de modélisation de profils d'apprenants, PMDLe, mis en œuvre dans un environnement unifié de gestion de profils, EPROFILEA. Cet environnement, qui s'appuie sur un ensemble de modèles, met à 
disposition des acteurs de l'apprentissage concernés, principalement les enseignants, des outils mutualisés d'exploitation de profils d'apprenants entièrement personnalisées, adaptées aux besoins pédagogiques des enseignants, mais aussi aux connaissances et compétences des apprenants, ainsi qu'aux capacités et préférences des utilisateurs concernés.

L'approche globale la plus analogue à la nôtre est celle adoptée dans le projet aLFanet (Santos et al., 2004) (aLFanet, 2005). La plateforme de formation à distance associée à ce projet aborde en effet à la fois des questions d'interopérabilité et des questions de personnalisation. Toutefois, l'interopérabilité n'est possible qu'avec d'autres plateformes compatibles du point de vue des normes pédagogiques respectées. Le projet se limite donc au monde IMS et n'est compatible, ni avec la majorité des EIAH, ni avec les approches papier-crayon. Quant à la personnalisation, elle concerne dans aLFanet uniquement les scénarios et les activités qu'ils comportent. aLFanet propose donc une approche intéressante, mais moins générique que la nôtre et qui va moins loin que la nôtre du point de vue de la personnalisation des activités.

Nous avons adopté dans nos travaux une démarche générique qui nous a conduites à proposer des modèles et processus génériques, que nous avons mis en œuvre dans des outils unifiés. Les utilisateurs de nos systèmes, principalement les enseignants, exploitent et personnalisent les modèles à travers les outils que nous mettons à leur disposition. Grâce à leur action sur les modèles qu'ils ont complétés et enrichis, ils disposent d'outils entièrement adaptés à leurs besoins et spécificités. Les enseignants sont ainsi au cœur de notre démarche. De plus, nous considérons que la place des enseignants dans la conception de nos systèmes perdure au-delà de la réalisation, dans les usages même des systèmes. Nous revendiquons que la généricité de notre approche n'est pas une limite à l'adaptation des outils à leurs utilisateurs finaux. Pour cela nous étudions l'articulation entre généricité de nos propositions et spécificités des besoins des utilisateurs. En effet, nous fournissons à l'utilisateur des outils génériques, sortes de boîtes vides qu'ils vont remplir, compléter et adapter aux spécificités de leurs besoins, en intégrant notamment une sémantique et des données à des outils qui en sont initialement dépourvus, achevant ainsi la conception des systèmes pour leur contexte particulier.

Ces travaux ouvrent des perspectives de recherche intéressantes concernant l'assistance aux utilisateurs d'applications informatiques. En effet, au cours des mises à l'essai que nous avons menées, nous avons pu constater que les outils opérationnalisant nos modèles sont difficiles à prendre en main et à utiliser. Une application telle que Regards propose en effet de nombreuses fonctionnalités nécessitant une manipulation de connaissances, qui peut s'avérer complexe, avec pour conséquence un risque que les utilisateurs renoncent à utiliser Regards malgré 
leur envie de profiter des possibilités offertes par cette application. Au-delà des nécessaires améliorations ergonomiques, l'ajout d'un système d'assistance permettrait d'éviter cet écueil. Une telle assistance devrait pouvoir s'adapter au contexte d'utilisation et aux spécificités de l'utilisation, telles que ses objectifs, son expérience et ses préférences en matière d'assistance.

\section{Références}

aLFanet, « Active Learning for Adaptive Internet », http://adenu.ia.uned.es/alfanet/, 2005.

Bull S., Mabbott A., « 20000 Inspections of a Domain-Independent Open Learner Model with Individual and Comparison Views », ITS'2006, Berlin, Germany, 2006a, p. 422-432.

Bull S., Mabbott A., « Student Preferences for Editing, Persuading, and Negotiating the Open Learner Model », ITS'2008, Berlin, Germany, 2006b, p. 481-490.

Bull S., Mabbott A., Abu-Issa A. S., « UMPTEEN: Named and Anonymous Learner Model Access for Instructors and Peers », IJAIED, vol 17, 2007, p. 227-253.

Bull S., Mangat M., Mabbott A., Abu-Issa A. S., Marsh J., « Reactions to Inspectable Learner Models: Seven Year Old to University Students », AIED'2005, Amsterdam, The Netherlands, 2005, p. 1-10

Bull S., McEvoy A. T., Reid E., « Learner Models to Promote Reflection in Combined Desktop PC / Mobile Intelligent Learning Environments », AIED'2003, Sydney, Australia, 2003, p. 199-208.

Bull S., McKay M., « An Open Learner Model for Children and Teachers: Inspecting Knowledge Level of Individuals and Peers », ITS'2004, Laceio, Brasil, 2004, p. 646-655.

Bull S., Pain H., « Did I say what I think I said, and do you agree with me?: Inspecting and questioning the student model ", World Conference on Artificial Intelligence in Education, Charlottesville, USA, 1995, p. 501-508.

Ginon B., Jean-Daubias S., « Models and tools to personalize activities on learners profiles », ED-MEDIA, 2011.

Ginon B., Jean-Daubias S., « Prise en compte des connaissances, capacités et préférences pour une personnalisation multi-aspects des activités sur les profils d'apprenants ", STICEF, vol 19, 2012.

Ginon B., Jean-Daubias S., Lefevre M., « Evolutive learners profiles », ED-MEDIA 2011, 2011.

Jean-Daubias S., «Ingénierie des profils d'apprenants », Habilitation à diriger les recherches, Université Claude Bernard Lyon 1, 2011.

Jean-Daubias S., Eyssautier-Bavay C., Lefevre M., « Modèles et outils pour rendre possible la réutilisation informatique de profils d'apprenants hétérogènes », STICEF, vol 16, 2009, p. 171-208.

Jean S., «PÉPITE : un système d'assistance au diagnostic de compétences », Thèse de doctorat, Université du Maine, 2000. 
28 Revue Technique et Science Informatique. Volume $\mathrm{X}-\mathrm{n}^{\circ} \mathrm{x} /$ année

Lazarinis F., Retalis S., « Analyze Me: Open Learner Model in an Adaptive Web Testing System », IJAIED, vol 17, 2007, p. 255-271.

Lefevre M., « Processus unifié pour la personnalisation des activités pédagogiques : métamodèle, modèles et outils », Thèse de doctorat en informatique, Université Claude Bernard Lyon 1, 2009.

Lefevre M., Jean-Daubias S., « Intégration de données hétérogènes : un exemple pour la constitution de profils d'apprenants », TICE, Lyon, France, 2012.

Lefevre M., Jean-Daubias S., Guin N., « PERSUA2, un modèle pour unifier le processus de personnalisation des activités d'apprentissage », EIAH'2011, 2011.

McCalla G., Bull S., Dimitrova V., « Special Issue (Part 1) - Open Learner Models: Research Questions », IJAIED, vol 17, 2007.

Mitrovic A., Martin B., «Evaluating the Effect of Open Student Models on SelfAssessment », IJAIED, vol 17, 2007, p. 121-144.

Nota_Bene, «Software for students results in private scholar establisments », www.omtviescolaire.fr/Gestion-privee-AEFE-Nota-Bene-Presentation.html, 2010.

Santos O. C., Barrera C., Boticario J., « An overview of aLFanet: an Adaptive iLMS based on standards », Eindhoven, The Netherlands, 2004, p. 429-432.

Self J., «Bypassing the intractable problem of student modelling », 1990, p. 107-123.

Van Rosmalen P., Vogten H., Van Es R., Passier H., Poelmans P., Koper R., « Authoring a full life cycle model in standards-based, adaptive elearning », vol 9, 2006, p. 72-83.

Vassileva J., McCall G., Greer J., «Multi-Agent Multi-User Modeling », vol 13, 2003, p. 179-210. 